\title{
RESEARCH
}

\section{Effect of regulatory warnings on antidepressant prescription rates, use of health services and outcomes among children, adolescents and young adults}

\author{
Laurence Y. Katz MD, Anita L. Kozyrskyj PhD, Heather J. Prior MSc, Murray W. Enns MD, \\ Brian J. Cox PhD, Jitender Sareen MD
}

$\infty$

See related article page 1025

\section{ABSTRACT}

Background: Regulatory bodies worldwide, including Health Canada, have issued warnings about prescribing antidepressants to children and adolescents. We sought to determine whether the Health Canada warning had the desired effects on prescribing patterns and outcomes and whether it had any unintended health consequences.

Methods: We examined data from prescription and health care databases representing more than 265000 children, adolescents and young adults annually to determine changes in the rates of antidepressant prescription, use of health services and outcomes in these populations in the 9 years before and the 2 years after the Health Canada warning. We also examined the data for unintended changes in these rates among patients with anxiety disorders. We used young adults as the comparison group because they were not targeted by the warning.

Results: Following the warning, the rate of antidepressant prescriptions decreased among children and adolescents (relative risk [RR] 0.86 , 95\% confidence interval $[\mathrm{Cl}]$ o.810.91 ) and among young adults (RR $0.90,95 \% \mathrm{Cl} 0.86-0.93$ ). Ambulatory visits because of depression decreased among children and adolescents (RR $0.90,95 \% \mathrm{Cl} 0.85-0.96$ ) and young adults (RR $0.91,95 \% \mathrm{Cl} 0.87-0.96$ ). The rate of completed suicides among children and adolescents rose significantly after the warning ( $R R 1.25,95 \% \mathrm{Cl} 1.08-1.44$; annual rate per $1000=0.04$ before and 0.15 after the warning). There was no equivalent change in the rate of completed suicides among young adults ( $R R$ 1.01, $95 \% \mathrm{Cl} 0.93^{-1.10}$; annual rate per $1000=0.15$ before and 0.22 after the warning). Among patients with an anxiety disorder, the prescription rates did not change among children and adolescents, except for a decrease in the use of selective serotonin reuptake inhibitors other than fluoxetine, but the rates among young adults changed similar to the pattern of changes in the overall prescribing of antidepressants. There was also a significant decrease in the rate of physician visits because of anxiety disorders among young adults after the warning.
Interpretation: Health advisories and warnings issued by regulatory bodies may have unintended consequences on the provision of care, delivery of health services and clinical outcomes. Further efforts are required to ensure that health warnings do not result in unexpected harm.

Une version française de ce résumé est disponible à l'adresse www.cmaj.ca/cgi/content/full/I78/8/1005/DCI

CMAJ 2008;178(8):1005-II

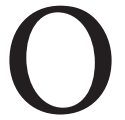

n June Io, 2003, the Committee on Safety of Medicines in the United Kingdom issued a warning against the use of paroxetine in children and adolescents. Health Canada and the US Food and Drug Administration issued further warnings, on June 3 and Oct. I5, 2004, respectively, about the use of all newer antidepressants (selective serotonin reuptake inhibitors and selective norepinephrine reuptake inhibitors) in children and adolescents. Similar warnings have been issued by many other regulatory agencies worldwide. ${ }^{1}$ These warnings were issued on the basis of results from randomized controlled trials showing that children and adolescents taking antidepressants were at greater risk of suicidal behaviour than those taking placebos. ${ }^{2}$

There are few data on the relation between regulatory action and outcomes, both in general and specifically for the warnings about antidepressant use in children and adolescents. Preliminary findings from the United Kingdom ${ }^{3}$ and the United States ${ }^{4-7}$ suggest that antidepressant prescribing decreased following the warnings in those jurisdictions. This has raised concerns about the relation between this regulatory action and the treatment of depression. ${ }^{5,8,9}$ In a study of the effect of regulatory action on benzodiazepine prescribing and hip fracture rates, Wagner and colleagues ${ }^{10}$ found no significant change in fracture rates after the regulatory action.

From the Department of Psychiatry (Katz, Enns, Cox, Sareen), University of Manitoba; and the Manitoba Centre for Health Policy, Department of Community Health Sciences (Kozyrskyj, Prior), University of Manitoba, Winnipeg, Man. 
We examined the rates of antidepressant prescription among children, adolescents and young adults in the province of Manitoba to determine whether the warning issued by Health Canada was associated with a decrease in the use of targeted medications. We also evaluated whether it had unintended consequences on the use of health services and on outcomes among patients with depression or anxiety disorders.

\section{Methods}

\section{Study population}

The province of Manitoba has a universal, single-payer health care system for medical services. The province covers the cost of prescribed medications for people who receive social assistance and for First Nations people. For all other residents, the medications are paid for either by the individual or by a private insurance plan. There is a provincial formulary that covers prescription costs above a threshold determined by the patient's income.

We included children (5-II years), adolescents (I2-I7 years) and young adults ( $19-24$ years) living in the province of Manitoba and registered with Manitoba Health from Apr. I, I995, to Mar. 3I, $2006(N=2920890)$. About $50 \%$ of the population was male, and about $50 \%$ lived in urban areas. The population that completed suicide had a significant overrepresentation of males and individuals from the rural north of Manitoba (Table I). Adolescents and young adults made up the vast majority of those who completed suicide. As a meas-

Table 1: Demographic characteristics of children, adolescents and young adults living in Manitoba and registered with Manitoba Health from Apr. 1, 1995, to Mar. 31, 2006

\begin{tabular}{|c|c|c|}
\hline \multirow[b]{2}{*}{ Characteristic } & \multicolumn{2}{|c|}{ No. (\%) of people } \\
\hline & $\begin{array}{c}\text { All } \\
n=2920890^{*}\end{array}$ & $\begin{array}{c}\text { Completed suicide } \\
n=235\end{array}$ \\
\hline \multicolumn{3}{|l|}{ Sex } \\
\hline Male & $1482220(50.8)$ & $175(74.5)$ \\
\hline Female & $1438670(49.3)$ & $60(25.5)$ \\
\hline \multicolumn{3}{|l|}{ Age, yr } \\
\hline $5-11$ & 1143281 (39.1) & $6 \quad(2.6)$ \\
\hline $12-17$ & 926632 (31.7) & $93(39.6)$ \\
\hline $19-24$ & 850977 (29.1) & $136(57.9)$ \\
\hline Residence location & & $n=159$ \\
\hline Urban & $1521162(52.1)$ & $57(35.8)$ \\
\hline Rural north & $246238 \quad(8.4)$ & $40(25.2)$ \\
\hline Rural south & 1153490 (39.5) & $62(39.0)$ \\
\hline \multicolumn{3}{|c|}{$\begin{array}{l}\text { Socioeconomic status; } \\
\text { income fifth, \$ }\end{array}$} \\
\hline 11 129-36 562 & $589958(20.2)$ & \\
\hline $36563-46767$ & $550088(18.8)$ & \\
\hline $46768-58645$ & 558302 (19.1) & \\
\hline $58646-73792$ & 597851 (20.5) & \\
\hline 73 793-279 150 & $614479(21.0)$ & \\
\hline
\end{tabular}

*This represents total number of children, adolescents and young adults during the 11-year study period. The annual mean number was 265535 . ure of socioeconomic status, we divided the study population into fifths by average household income from 200I Canadian census data (Table I).

The study was approved by the Human Research Ethics Board, University of Manitoba, and Manitoba's Health Information and Patient Confidentiality Committee.

\section{Data sources}

We obtained data from 4 electronic databases maintained by the Manitoba Health Services Insurance Plan: registry files, physician reimbursement claims, hospital discharge abstracts and records of prescriptions dispensed. The registry files contain records for every resident of Manitoba, and each record includes date of birth, sex and geographic location. Physician reimbursement claims contain information about each diagnosis. Until Mar. 3I, 2004, discharge abstracts for hospital services included information for up to 16 diagnosis codes selected from the International Classification of Diseases, 9th revision, clinical modification (ICD-9-CM). Since then, the discharge abstracts include up to 25 diagnosis codes selected from the International Statistical Classification of Diseases and Related Health Problems, Ioth revision (ICDIo). All records of dispensed prescriptions are submitted by retail pharmacies and contain information about the date of dispensing, the drug name and identification number, the dosage form and the quantity dispensed. ${ }^{11}$ The reliability and validity of the Manitoba Health Services Insurance Plan's prescription databases are high (i.e., the prescription database captures $93 \%$ of dispensed prescriptions and there is $92 \%$ agreement between the database and the written prescription)..$^{12,13}$ The success of database linkage at the Manitoba Centre for Health Policy is very high, approaching 100\%. ${ }^{14}$

We obtained data on completed suicides from Manitoba's vital statistics files, the chief medical examiner's office and the College of Physicians and Surgeons of Manitoba. We reviewed 2 years of suicide data to evaluate the consistency of the designation of completed suicide by the different sources. We found the consistency to be very high (97.8\%). Suicide data for the province are in line with data for the rest of Canada and the United States, ${ }^{15}$ which suggests generalizability of the results of this study.

\section{Antidepressant prescriptions}

For our analysis, we identified all episodes of antidepressant prescription over the study period among I I43 28I children (annual mean I03 934), 926632 adolescents (annual mean 84239 ) and 850977 young adults (annual mean 77 362) that met the following criteria: outpatient antidepressant prescription filled between Apr. I, 1995, and Mar. 3I, 2006; and ICD-9-CM diagnosis code 296,300 or 3 II. To evaluate whether Health Canada's warning was associated with changes in the treatment of anxiety disorders (not targeted by the warning), we performed a subanalysis using only ICD-9CM code 300.

We identified prescriptions for antidepressants using the Anatomic Therapeutic Chemical Classification system and generic drug names. We grouped antidepressants as follows: tricyclic antidepressants (amitriptyline, nortriptyline, desipra- 
mine and imipramine); fluoxetine; other selective serotonin reuptake inhibitors (paroxetine, citalopram, fluvoxamine and sertraline); and other antidepressants (venlafaxine, mirtazapine and nefazadone). We singled out fluoxetine in the analysis because of recommendations from psychiatric organizations indicating that its risk-benefit profile was favourable given its efficacy data. The other antidepressants did not have equivalent efficacy data and thus were determined not to be first-line treatments by these organizations based on their risk-benefit profiles.

\section{Statistical analysis}

We calculated the prevalence (and 95\% confidence intervals [CIs]) of antidepressant prescriptions in each fiscal year from Apr. I, 1995, to Mar. 3I, 2004, and from Apr. I, 2004, to Mar. 3I, 2006. We selected young adults as the comparison group because the Health Canada warning was directed at children and adolescents aged 17 years or less. We excluded adolescents aged I8 years from our analysis owing to concerns that they would bias the intervention and comparison groups. We analyzed the annual prevalence rates of antidepressant prescriptions based on age, sex, residence location and socioeconomic status using a generalized linear model framework, with either a Poisson or negative binomial distribution according to whichever distribution provided the best model fit. We modelled antidepressant prescription as a function of linear or quadratic time and the other explanatory variables. We selected the linear time model as the best model (deviance statistic closest to I) based on the deviance of the model fit statistic divided by the degrees of freedom. Because of the longitudinal nature of the data, we used generalized estimating equation $\mathrm{s}^{16}$ to account for the correlation structure. We calculated contrast estimates of the change in slope of antidepressant prescriptions after the Health Canada warning and tested whether the change over time was significant. We included a time-by-age-group-by-warning interaction in the model to determine whether changes in the trends were statistically significant between age groups. We report the relative risks (RRs) and 95\% CIs for the change in the slope of the linear trend over time (obtained by exponentiation of the contrast estimates). The models selected were the most parsimonious for the data structure.

For the analysis of health services use and outcomes, we used the diagnostic codes in Box I to identify episodes of health services use (physician office visit, hospital admission) and outcomes (admission to hospital because of suicide attempt, completed suicide) among children, adolescents and young adults. For the analysis of suicide, we included people who died between Jan. I, I995, and Dec. 31, 2005; we excluded children less than 8 years old because there were no suicides in this age group. We calculated the annual rates per Iooo people of hospital admissions and physician office visits for any diagnosis or suicidal behaviour with a diagnostic code in Box I for each fiscal year from Apr. I, I995, to Mar. 3I, 2004, and from Apr. I, 2004, to Mar. 3I, 2006. In addition, we calculated the annual rates of completed suicide among children and adolescents (8-I7 years) and among young adults (I9-24 years). We modelled the rates of health services use
Box 1: Diagnostic codes used to identify health services use and outcomes related to depression and anxiety disorders*

Health services use

Physician office visit

- ICD-9-CM codes: 296,300 or 311

Hospital admission

- ICD-9-CM codes: 296.20, 296.23, 296.24, 296.30, 296.31, 296.32, 296.33, 296.34, 296.36, 296.51, 296.53, 296.90, 296.99, 300.4, 311

- ICD-10 codes: F31.3, F31.4, F31.5, F32-F33.9, F34.1, F34.8, F34.9, F38.1, F38.8, F39, F41.2, F53

Suicide attempt

- ICD-9 CM codes E950 to E959 and ICD-10 codes X60-X84 Completed suicide

- ICD-9 CM codes E950 to E959 and ICD-10 codes X60-X84

Note: ICD-9-CM = International Classification of Diseases, 9th revision, clinical modification; ICD-10 = International Statistical Classification of Diseases and Related Health Problems, 10th revision.

*Health services included physician office visits, and hospital admissions because of depression and anxiety disorders; outcomes included admissions to hospital because of suicide attempt, and completed suicide.

and outcomes using regression analyses as described earlier. We compared changes over time in health services use and suicide outcomes among children and adolescents with changes over time among young adults.

\section{Results}

\section{Antidepressant prescription rates}

The number of individuals prescribed an antidepressant during the II-year study period was 452I children (annual mean 4II), I6 79I adolescents (annual mean 1526) and 37065 young adults (annual mean 3370).

Before the Health Canada warning was issued, overall prescriptions of antidepressants were on the rise among children and adolescents and among young adults (Figure I). Following the warning, the prescription rate decreased significantly in both age groups (children and adolescents: RR 0.86, 95\% CI $0.8 I-0.9 I$; young adults: RR $0.90,95 \%$ CI $0.86-0.93$ ) (Table 2). The change in rates did not differ significantly between the 2 groups $(p=0.2 \mathrm{I})$. Among those with a diagnosis of an anxiety disorder, the rate of antidepressant prescription also decreased; this decrease was not significant among children and adolescents (RR o.88, 95\% CI 0.75-I.03), but it was significant among young adults (RR $0.84,95 \% \mathrm{CI} 0.75-0.94$ ). The change in rates did not differ significantly between the 2 groups $(p=0.65)$.

Fluoxetine use remained relatively constant among both children and adolescents but showed a downward trend among young adults for a number of years before the Health Canada warning was issued (Figure 2). After the warning, the rate of fluoxetine use increased significantly among children and adolescents (RR I.IO, 95\% CI I.02-I.I9) and among young adults (RR I.23, 95\% CI I.IO-I.38) (Table 2, Figure 2). The change in rates did not differ significantly between the 


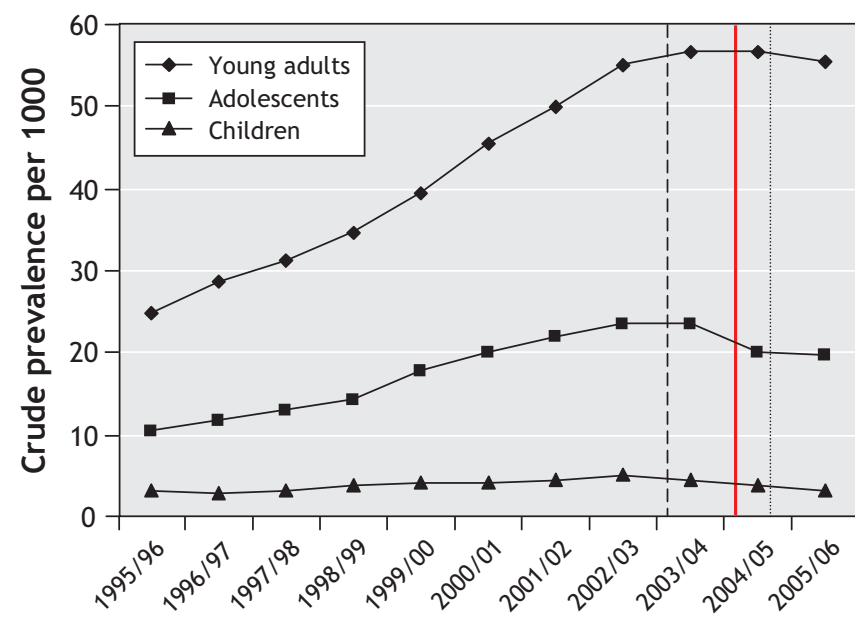

Figure 1: Crude annual prevalence of antidepressant prescriptions per 1000 children (5-11 years), adolescents (12-17 years) and young adults (19-24 years) in the province of Manitoba, by fiscal year, before and after Health Canada issued a warning about antidepressant use in children and adolescents (date indicated by red line). People aged 18 years were excluded because of an unclear impact of the warning on prescribing in this age group. The dates are indicated for similar warnings issued in the United Kingdom (dashed line) and the United States (dotted line).

2 groups was not significant $(p=0.12)$. Among those with a diagnosis of an anxiety disorder, the rate of fluoxetine use also increased; the increase was not significant among children and adolescents (RR I.I3, 95\% CI 0.94-I.37), but it was significant among young adults (RR I.65, 95\% CI I.IO-2.46). The change in rates did not differ significantly between the 2 groups $(p=0.10)$.

The use of selective serotonin reuptake inhibitors other than fluoxetine decreased significantly after the Health Canada warning among children and adolescents (RR 0.68 , $95 \% \mathrm{CI} 0.63-0.73$ ) and among young adults (RR $0.80,95 \%$ CI $0.75-0.85$ ) (Table 2). The change in rates was significantly greater among the children and adolescents than among the young adults $(p<0.001)$. Among those with a diagnosis of an anxiety disorder, the prescription rate also decreased significantly in both groups (children and adolescents: RR 0.62, 95\% CI 0.50-0.76; young adults: RR 0.78, $95 \%$ CI $0.73-0.85$ ). The change in rate was significantly greater among the children and adolescents than among the young adults $(p=0.03)$.

The prescription rates of other antidepressants decreased significantly after the warning among children and adolescents (RR $0.59,95 \%$ CI $0.50-0.69$ ) and among young adults (RR 0.82, 95\% CI 0.75-0.89) (Table 2). The rates declined to a significantly greater extent among children and adolescents than among young adults $(p<0.00 \mathrm{r})$. Among those with an anxiety disorder, the prescription rate also decreased; the decrease was not significant among children and adolescents (RR $0.77,95 \%$ CI 0.46-I.28), but it was significant among young adults (RR 0.69, 95\% CI 0.59-0.8I). The change in rates did not differ significantly between the 2 groups $(p=0.70$ ).

\section{Health services use}

The rate of physician visits because of depression decreased significantly after the warning among children and adolescents (RR 0.90, 95\% CI 0.85-0.96) and among young adults (RR 0.9I, 95\% CI 0.87-0.96). The change in rates did not differ significantly between the 2 groups $(p=0.79)$. There was no significant change in the rate of physician visits because of anxiety disorders after the warning among children and adolescents (RR 0.95, 95\% CI 0.89-I.02), but there was a significant decrease among young adults (RR 0.95, 0.90-0.99). The difference in the change in rates between the 2 groups was not significant $(p=0.93)$.

The rate of hospital admissions because of depression did not change after the warning in either group.

\section{Suicide}

Between 1995 and 2005, there were 99 completed suicides among children and adolescents and 136 among young adults. The rate of completed suicide among children and adolescents increased significantly after the Health Canada warning was issued (RR I.25, 95\% CI I.08-I.44; annual rate per I000 = 0.04 before and 0.15 after the warning). There was no significant change in the rate of completed suicide among young adults after the warning (RR I.oI, 95\% CI 0.93-I.IO; annual rate per I000 $=0.15$ before and 0.22 after the warning). The change in rates differed significantly between the young adults and the children and adolescents $(p<0.02)$ (Figure 3$)$. The rate of suicide attempts did not change significantly after the warning among children and adolescents (RR 0.84, 95\% CI 0.63-I.I2) or among young adults (RR $0.76,95 \%$ CI $0.57-$ I.03).

Table 2: Relative risk of receiving an antidepressant prescription after Health Canada issued a warning about the use of antidepressants in children and adolescents, by age group and type of antidepressant

Type of antidepressant; relative risk $(95 \% \mathrm{Cl})$

\begin{tabular}{lcccc}
\cline { 2 - 5 } Group & All antidepressants* & Fluoxetine* $^{*}$ & $\begin{array}{c}\text { Other selective serotonin } \\
\text { reuptake inhibitors* }\end{array}$ & Other antidepressants ${ }^{*}$ \\
\hline Children and adolescents & $0.86(0.81-0.91)$ & $1.10(1.02-1.19)$ & $0.68(0.63-0.73) \neq$ & $0.59(0.50-0.69) \neq$ \\
Young adults & $0.90(0.86-0.93)$ & $1.23(1.10-1.38)$ & $0.80(0.75-0.85)$ & $0.82(0.75-0.89)$ \\
\hline
\end{tabular}

Note: $\mathrm{Cl}=$ confidence interval, SSRI = selective serotonin reuptake inhibitor

*Determined by means of negative binomial regression modelling.

†Determined by means of Poisson regression modelling.

$\neq p<0.001$ compared with young adults. 
Of the children and adolescents who completed suicide, Io\% had received an antidepressant prescription in the 9 years before the warning, $10.5 \%$ had received a prescription in the first year after the warning, and $4 \%$ had received a prescription in the second year after the warning. These percentages were not statistically different from each other $(p=0.94)$. There was no change in fluoxetine use after the warning among those who completed suicide.

The most common method of suicide was hanging. This method was used in $96 \%$ of the deaths by suicide in 2005 . Firearms and poisoning were less frequent methods. Among children and adolescents who completed suicide between 2003 and 2005, the average age was between I4.3 and I5.8 years (range $8-17$ years); $37 \%-48 \%$ were female and $52 \%-$ $63 \%$ were male. The percentage of people who completed suicide who were Aboriginal remained relatively consistent ( $74 \%-85 \%)$, and the percentage of on-reserve deaths decreased from 73 to 57 between 2003 and 2005. There was no evidence of clustering of suicides according to either time or residence location.

\section{Interpretation}

We undertook a system-wide evaluation of the treatment of depressive and anxiety disorders in children and adolescents in Manitoba in the 9 years before and the 2 years following the Health Canada warning about the use of antidepressants in this age group. Our findings suggest that the warning not only affected prescribing patterns of the drugs mentioned in the warning but that it also had spillover effects on the provision of care for indications and populations not targeted by the warning. We found a $14 \%$ decrease in the rate of antidepressant prescription among children and adolescents after the warning. The rates of use of newer antidepressants, excluding fluoxetine, decreased by $32 \%-40 \%$. Fluoxetine use increased by $10 \%$ in the period following the warning. During the same period, the rate of antidepressant prescription decreased by 10\% among young adults, a patient population that was not targeted by the warning. In addition, we found that the rate of physician visits because of depression decreased in both age groups. This finding indirectly suggests that psychotherapy (at least as provided by physicians) did not replace the absent pharmacotherapy.

We found that the rate of completed suicides among children and adolescents increased by $25 \%$ following the warning. There was no such change among young adults. We found no change in the rates of suicide attempts that required hospital admission or in the rates of hospital admission because of depression among either children and adolescents or young adults.

In our subanalysis we found that the Health Canada warning was not associated with changes in antidepressant prescription rates among children and adolescents with anxiety disorders, except for a decrease in the prescription of selective serotonin reuptake inhibitors other than fluoxetine. Similarly, there was no significant change in the rate of physician visits among children and adolescents with anxiety disorders in the period after the warning. Among young adults with

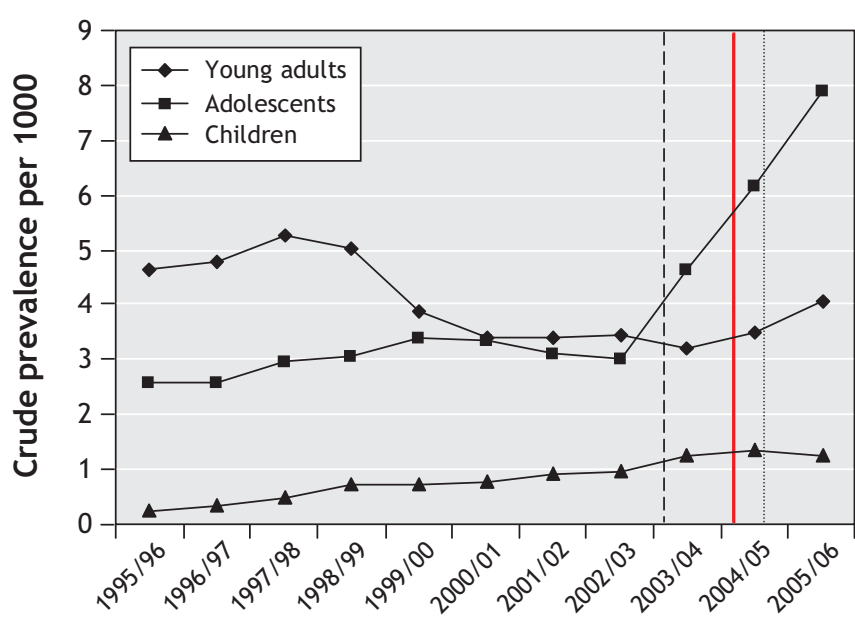

Figure 2: Crude annual prevalence of fluoxetine prescriptions per 1000 children (5-11 years), adolescents (12-17 years) and young adults (19-24 years) in the province of Manitoba, by fiscal year, before and after Health Canada issued a warning about antidepressant use in children and adolescents (date indicated by red line). People aged 18 years were excluded because of an unclear impact of the warning on prescribing in this age group. The dates are indicated for similar warnings issued in the United Kingdom (dashed line) and the United States (dotted line).

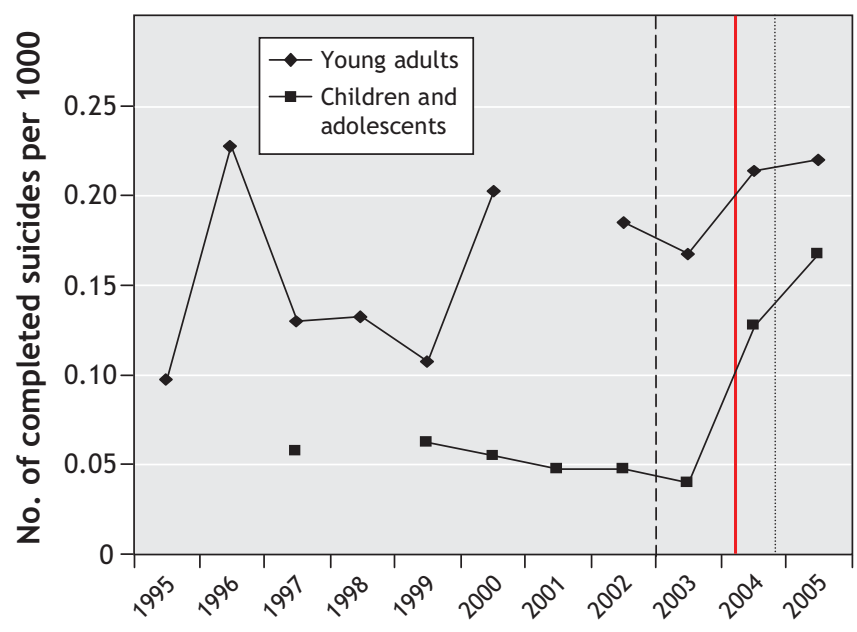

Figure 3: Crude annual rates of completed suicide per 1000 children and adolescents (8-17 years) and young adults (19-24 years), by year, before and after Health Canada issued a warning about antidepressant use in children and adolescents (date indicated by red line). People aged 18 years were excluded because of an unclear impact of the warning on prescribing in this age group. Data for years with fewer than 6 suicides, although included in the outcome analysis, are not portrayed in the figure for confidentiality purposes. The dates are indicated for similar warnings issued in the United Kingdom (dashed line) and the United States (dotted line). 
anxiety disorders, however, we found that the rate of antidepressant prescription was similar to the pattern of changes in the overall prescribing of antidepressants. There was also a significant decrease in the rate of physician visits because of anxiety disorders among young adults after the warning.

Our study raises several questions about the impact of warnings from regulatory bodies. First, as in other studies, ${ }^{1,3-5}$ we found a decrease in the rates of antidepressant prescription both among children and adolescents and among young adults in the period following the Health Canada warning. The regulatory bodies stated that their intent was not to discourage prescribing; ${ }^{17}$ nevertheless it did occur in association with the warnings. Furthermore, we found decreased prescribing among young adults, a population that was not targeted by the Health Canada warning. This supports the findings of Valuck and colleagues, ${ }^{8}$ who demonstrated similar spillover effects. Second, we found a decrease in the rate of physician visits because of depressive disorders among children, adolescents and young adults as well as a decrease in the rate of visits because of anxiety disorders among young adults. These findings are similar to those of other studies that documented decreases in treatment episodes for depression in children and adolescents and young adults in association with regulatory warnings in the United States. ${ }^{8,9}$ It is of concern that, in an environment where the treatment of a life-threatening disorder has become more complicated, vulnerable patients are receiving less care. Richardson and colleagues ${ }^{18}$ reported that attitudes of health care providers toward the treatment of depression in adolescents have not changed, which suggests that the changes in the treatment of depression observed in the United States may have been related to patient and family variables. It may also be that the warnings and the media response invoked fear of diagnosis and treatment among patients and their families. ${ }^{19}$ Furthermore, Richardson and colleagues ${ }^{18}$ found that providers had not changed their practices to meet the guidelines regarding monitoring of patients recommended in association with the warnings. This is similar to the impact of regulatory action on prescribing practices in the use of cyclooxygenase- 2 inhibitors. Following the withdrawal of rofecoxib, there was a decrease in prescribing of cyclooxygenase-2 inhibitors; however, physicians did not follow the recommended guidelines for alternative treatments. ${ }^{20,21}$

The increased rate of suicide that we observed among children and adolescents in the period following the Health Canada warning is of concern, especially because it coincided with a decrease in the rate of physician visits for the treatment of depressive and anxiety disorders. Our finding is similar to that of Gibbons and colleagues, ${ }^{5}$ who reported an association between the regulatory warning in the United States and an increased suicide rate among children and adolescents. We are unable to draw conclusions about whether the decrease in treatment of depressive and anxiety disorders caused the increase in suicides; we can merely document an association between the regulatory warning and an increase in the suicide rate. Factors other than antidepressant prescribing may be responsible for the observed changes. Fur- thermore, only 2 years of data were available after the warning for our analysis, and conclusions on whether this finding indicates a trend or an anomaly would be premature. Collectively, however, our observations raise concerns over the potential for the regulatory warning to lead to untreated depression and other related mental health issues. The vast majority of children and adolescents who completed suicide had not received an antidepressant before the time of death, a finding supported by a review of autopsy results for the children and adolescents who completed suicide after the warning (unpublished data). This finding is also consistent with results from other studies of suicide among youth taking antidepressants. ${ }^{22-24}$

We postulate that the observed changes following the warning would not be confined to a single Canadian jurisdiction or regulatory authority. Indeed, other studies have consistently documented changes in the use of antidepressants, but none has documented such a wide range of unintended health consequences.

Our study has limitations beyond those described above. First, physician coding of diagnoses may not have been accurate in all cases, and the prescription data may not have reflected actual use. Second, the validity of the diagnosis of suicide is impossible to ascertain, a problem inherent to the study of suicide.

In conclusion, the warning issued by Health Canada about the use of antidepressants in children and adolescents was associated with a decrease in the use of the targeted medications. However, the warning had important and unintended adverse consequences on the delivery of health services to patients with depressive and anxiety disorders, both those targeted and not targeted by the warning. Given the potentially devastating consequences of some health warnings, greater attention must be paid to optimal dissemination strategies for health advisories and warnings. Regulatory bodies should conduct timely evaluations of major health warnings to ensure that they have the desired effects and, if not, to institute appropriate corrective measures.

This article has been peer reviewed.

Competing interests: Jitender Sareen is a member of the speakers bureau of Wyeth, Astra Zeneca and Bioval. None declared for Laurence Katz, Anita Kozyrskyj, Heather Prior, Murray Enns or Brian Cox.

Contributors: Laurence Katz had full access to all of the study data and takes responsibility for the integrity of the data and the accuracy of its analysis. All of the authors contributed to the design and conduct of the study. Laurence Katz, Anita Kozyrskyj and Heather Prior performed the data collection and, with Murray Enns, Brian Cox and Jitender Sareen, analyzed and interpreted the data. All of the authors participated in the preparation of the manuscript, revised it critically for important intellectual content and approved the version submitted for publication.

Acknowledgements: We thank Dr. Dan Chateau, Biostatistics Unit, University of Manitoba, for his consultation on the statistical methods used in the study. He received a consultant's fee for his assistance.

This study was supported by a grant from the Health Sciences Centre Foundation awarded to Laurence Katz. It was also supported by a Tier II Canada Research Chair Award to Brian Cox and by a Canadian Institutes for Health Research New Investigator Award to Jitender Sareen.

The results and conclusions are those of the authors, and no official endorsement by Manitoba Health is intended or should be inferred. 


\section{REFERENCES}

I. Kurdyak PA, Juurlink DN, Mamdani MM. The effect of antidepressant warnings on prescribing trends in Ontario, Canada. Am J Public Health 2007;97:750-4

2. Hammad TA, Laughren T, Racoosin J. Suicidality in pediatric patients treated with antidepressant drugs. Arch Gen Psychiatry 2006;63:332-9.

3. Murray ML, Thompson M, Santosh PJ, et al. Effects of the Committee on Safety of Medicines advice on antidepressant prescribing to children and adolescents in the U.K. Drug Saf 2005;28:115I-7.

4. Nemeroff CB, Kalali A, Keller MB, et al. Impact of publicity concerning pediatric suicidality data on physician practice pattern in the United States. Arch Gen Psychiatry 2007;64:466-72.

5. Gibbons RD, Brown $\mathrm{CH}$, Hur K, et al. Early evidence on the effects of regulators suicidality warnings on SSRI prescriptions and suicide in children and adolescents. Am J Psychiatry 2007;164:1356-63.

6. Kurian BT, Ray WA, Arbogast PG, et al. Effect of regulatory warnings on antidepressant prescribing for children and adolescents. Arch Pediatr Adolesc Med 2007;16r:690-6.

7. Olfson M, Marcus SC, Druss BG. Effects of food and drug administration warnings on antidepressant use in a national sample. Arch Gen Psychiatry 2008;65:94-Ior

8. Valuck RJ, Libby AM, Orton HD, et al. Spillover effects on treatment of adult depression in primary care after FDA advisory on risk of pediatric suicidality with SSRIs. Am J Psychiatry 2007;164:1198-205.

9. Libby AM, Brent DA, Morrato EH, et al. Decline in treatment of pediatric depression after FDA advisory on risk of suicidality with SSRI's. Am J Psychiatry 2007;I64:884-9I.

Io. Wagner AK, Ross-Degnan D, Gurwitz JH, et al. Effect of New York state regulatory action on benzodiazepine prescribing and hip fracture rates. Ann Intern Med 2007; I46:96-103.

II. Kozyrskyj AL, Carrie AG, Mazowita GB, et al. Decrease in antibiotic use among children in the I990s: not all antibiotics, not all children. CMAJ 2004;171:133-8.

I2. Kozyrskyj AL, Mustard CA. Validation of an electronic, population-based prescription database. Ann Pharmacother 1998;32:1152-7.

I3. Robinson JR, Young TK, Roos LL, et al. Estimating the burden of disease. Comparing administrative data and self-reports. Med Care 1997;35:932-47.

I4. Roos LL, Nicol JP. A research registry: uses, development and accuracy. J Clin Epidemiol I999;52:39-47.

I5. Martens P, Fransoo R, McKeen N, et al. Manitoba Centre for Health Policy. Patterns of regional mental illness disorder diagnoses and service use in Manitoba: a population-based study. Winnipeg: Manitoba Health;2004. p. 313-352.

I6. Edwards LJ. Modern statistical techniques for the analysis of longitudinal data in biomedical research. Pediatr Pulmonol 2000;30:330-44.

17. Leon AC. The revised warning for antidepressants and suicidality: unveiling the black box of statistical analyses. Am J Psychiatry 2007;164:1786-9.

I8. Richardson LP, Lewis CW, Casey-Goldstein M, et al. Pediatric primary care providers and adolescent depression: a qualitative study of barriers to treatment and the effect of the black box warnings. JAdolesc Health 2007;40:433-9.

19. Pfeffer CR. The FDA pediatric advisories and changes in diagnosis and treatment of pediatric depression. Am J Psychiatry 2007;164:843-6.

20. Williams D, Singh $M$, Hind C. The effect of the withdrawal of rofecoxib on prescribing patterns of COX-2 inhibitors in Scotland. Br J Clin Pharmacol 2006;62:366-8.

2I. Barozzi N, Tett SE. What happened to the prescribing of other COX-2 inhibitors paracetemol and non-steroidal anti-inflammatory drugs when rofecoxib was withdrawn in Australia? Pharmacoepidemiol Drug Saf 2007;16:II84-9I.

22. Isacsson G, Homgren P, Ahlner J. Selective serotonin reuptake inhibitor antidepressants and the risk of suicide: a controlled forensic database study of $\mathrm{I}_{4}, 857$ suicides. Acta Psychiatr Scand 2005; III:286-90.

23. Leon AC, Marzuk PM, Tardiff K, et al. Paroxetine, other antidepressants, and youth suicide in New York City: 1993 through 1998. J Clin Psychiatry 2004;65:915-8.

24. Sondergard L, Kvist K, Andersen PK, et al. Do antidepressants precipitate youth suicide? Eur Child Adolesc Psychiatry 2006;15:232-40.

Correspondence to: Dr. Laurence Katz, Department of Psychiatry,

University of Manitoba, Rm. PZ-I62, 77 I Bannatyne Ave.,

Winnipeg MB R3E 3N4; fax 204 787-4975; lkatz@hsc.mb.ca 